\title{
Highly Oriented and Ordered Water Soluble Semiconducting Polymers in a DNA Matrix
}

\author{
Moon Jong Han ${ }^{\dagger}$, Michael McBride ${ }^{\ddagger}$, Bailey Risteen ${ }^{\star}$, Guoyan Zhang ${ }^{\ddagger}$, Brian V. Khau ${ }^{\star}$, Elsa \\ Reichmanis $^{\ddagger},, \|, *$, and Dong Ki Yoon ${ }^{\dagger, \perp, *}$
}

*To whom correspondence should be addressed.

E-mail: nandk@kaist.ac.kr and ereichmanis@chbe.gatech.edu

${ }^{\dagger}$ Graduate School of Nanoscience and Technology, Korea Advanced Institute of Science and Technology, Daejeon 34141, Republic of Korea

\#School of Chemical and Biomolecular Engineering, Georgia Institute of Technology, Atlanta, GA 30332, USA

${ }^{\S}$ School of Chemistry and Biochemistry, Georgia Institute of Technology, Atlanta 30332, USA

"School of Materials Science and Engineering, Georgia Institute of Technology, Atlanta 30332, USA

${ }^{\perp}$ Department of Chemistry and KINC, Korea Advanced Institute of Science and Technology, Daejeon 34141, Republic of Korea 


\section{Table of Contents}

S1. Percentage of aggregates calculated by Frank-Condon fitting

S2. Absorbance spectra with polarized light

S3. POM images with the retardation plate. $(\lambda=530 \mathrm{~nm})$

S4. AFM for thickness measurement

S5. XPS measurement

S6. Output characteristics for different concentration

S7. Transfer characteristics of spin-coated films for different concentration condition

S8. Forward and backward transfer curves for spin-coated P3PHT film and P3PHT+DNA film

S9. Contact resistance measurement depending on DNA concentrations

S10. Forward and backward transfer curves of P3PHT, P3PHT $+\mathrm{Cu}^{2+}$, and P3PHT $+\mathrm{Cu}^{2+}-$ DNA films 


\section{S1. Percentage of aggregates calculated by Frank-Condon fitting.}

(a)

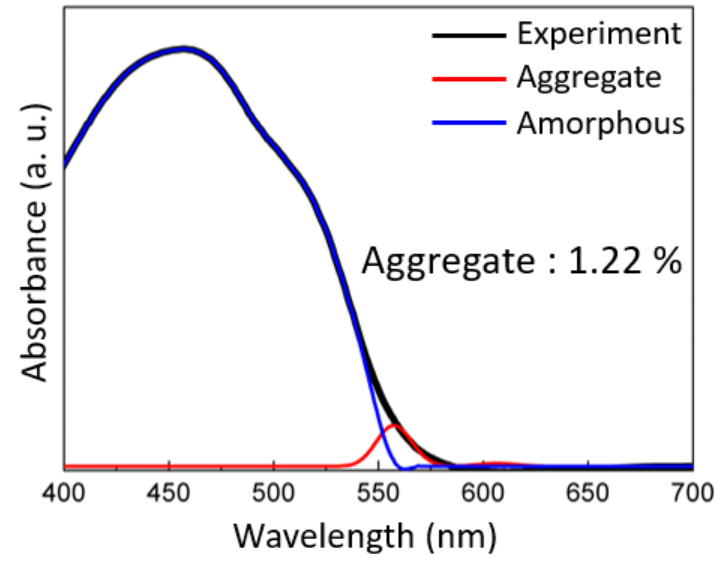

(c)

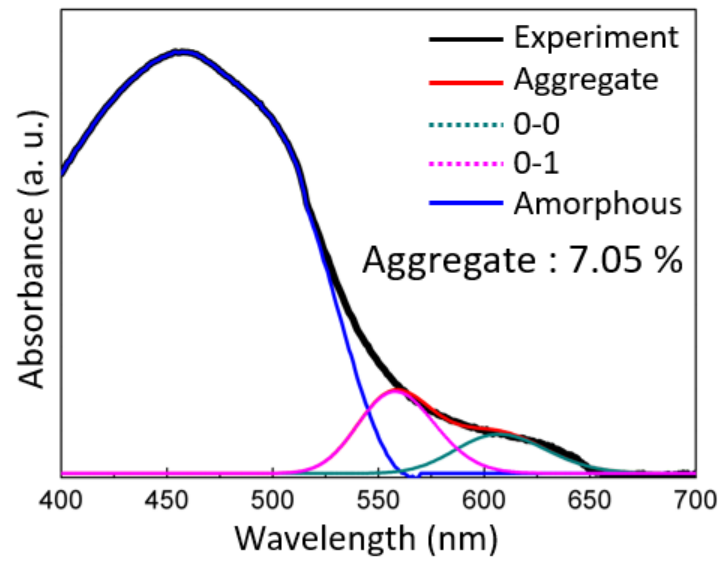

(b)

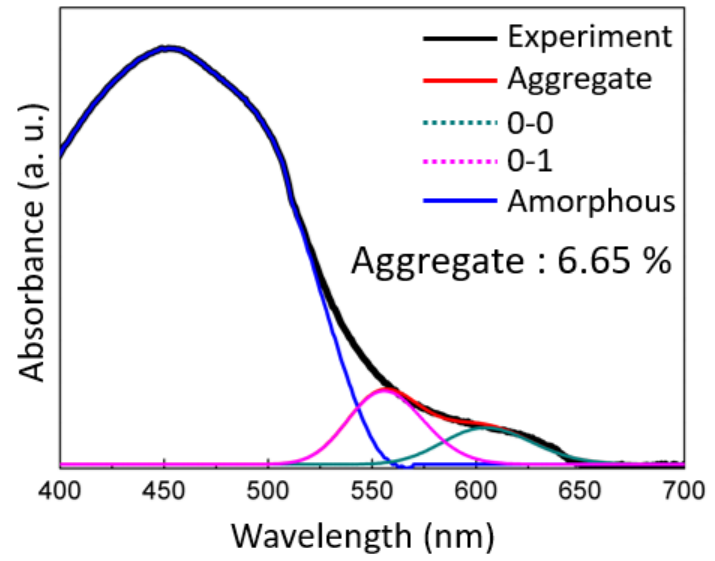

(d)

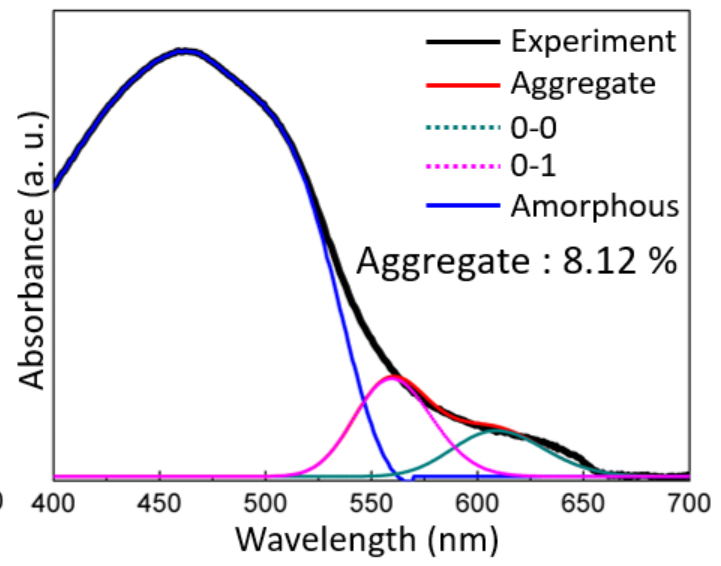

Figure S1. Frank-Condon fitting of UV-Vis absorption spectra for obtaining percent aggregates in P3PHT/DNA solution with different concentrations such as (a) P3PHT (15 mg/mL), (b) P3PHT $(15 \mathrm{mg} / \mathrm{mL})+$ DNA $(1.5 \mathrm{mg} / \mathrm{mL})$, (c) P3PHT (15 mg/mL) + DNA (3 mg/mL), and (d) P3PHT (15 $\mathrm{mg} / \mathrm{mL})+\operatorname{DNA}(7.5 \mathrm{mg} / \mathrm{mL})$. 


\section{S2. Absorbance spectra with polarized light}

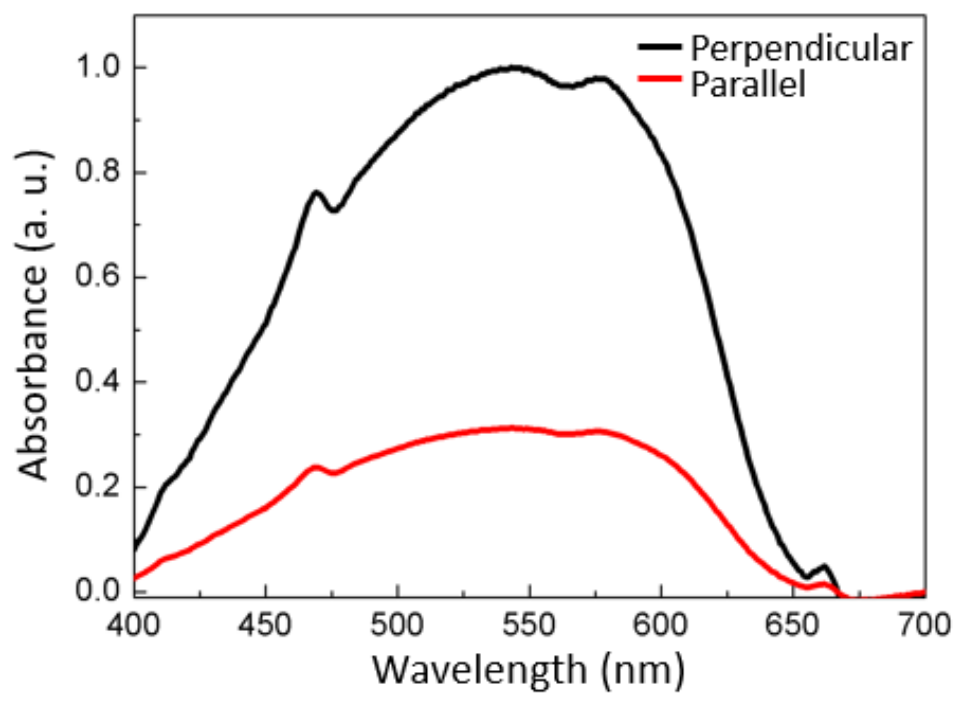

Figure S2. Absorbance spectra of aligned film of P3PHT $(15 \mathrm{mg} / \mathrm{mL})+$ DNA $(3 \mathrm{mg} / \mathrm{mL})$ concentration condition with the light source being polarized parallel or perpendicular to the shearing direction. 
S3. POM images with the retardation plate. $(\lambda=530 \mathrm{~nm})$

(a)

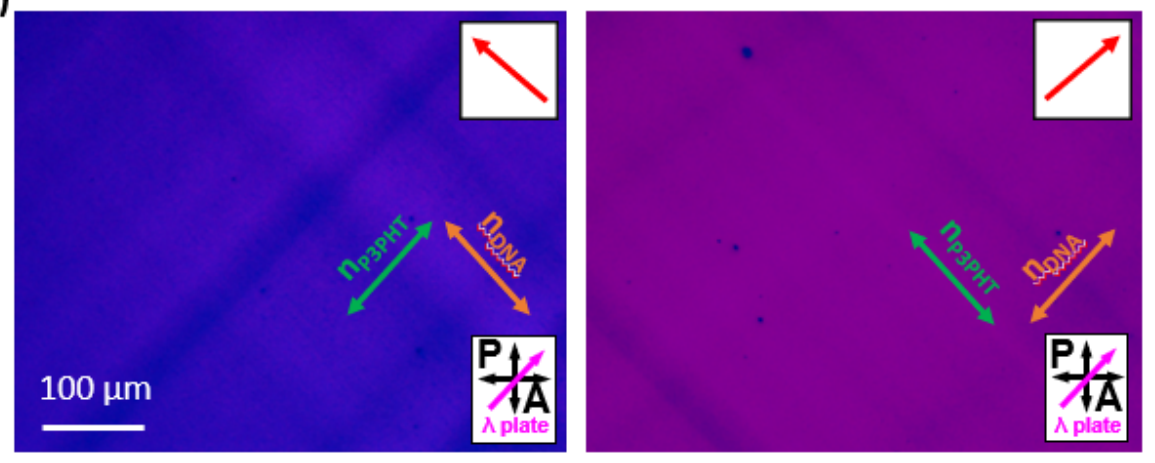

(b)

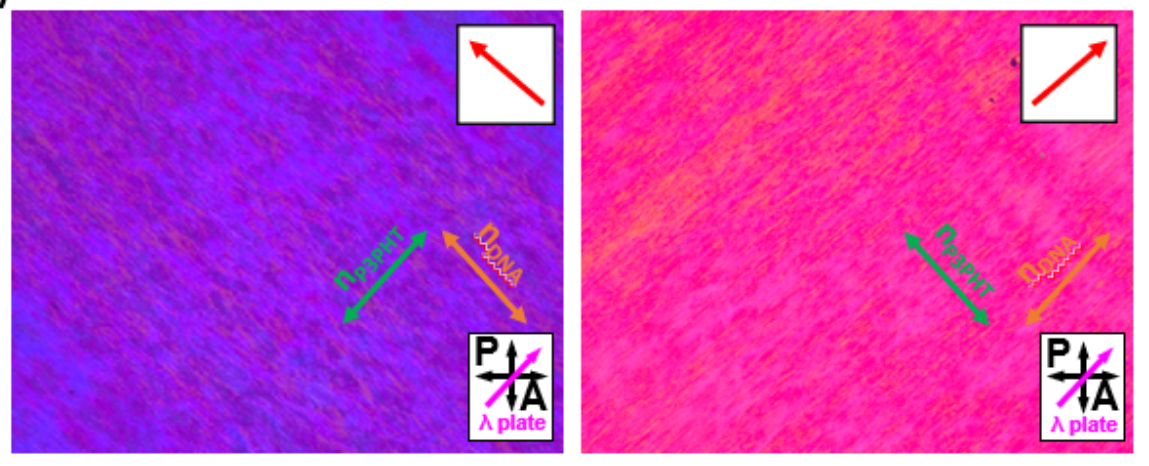

Figure S3. The orientation of P3PHT/DNA films of (a) P3PHT $(15 \mathrm{mg} / \mathrm{mL})+$ DNA $(1.5 \mathrm{mg} / \mathrm{mL})$, (b) P3PHT $(15 \mathrm{mg} / \mathrm{mL})+$ DNA $(7.5 \mathrm{mg} / \mathrm{mL})$ concentration condition was confirmed by POM images with the retardation plate. 
S4. AFM for thickness measurement
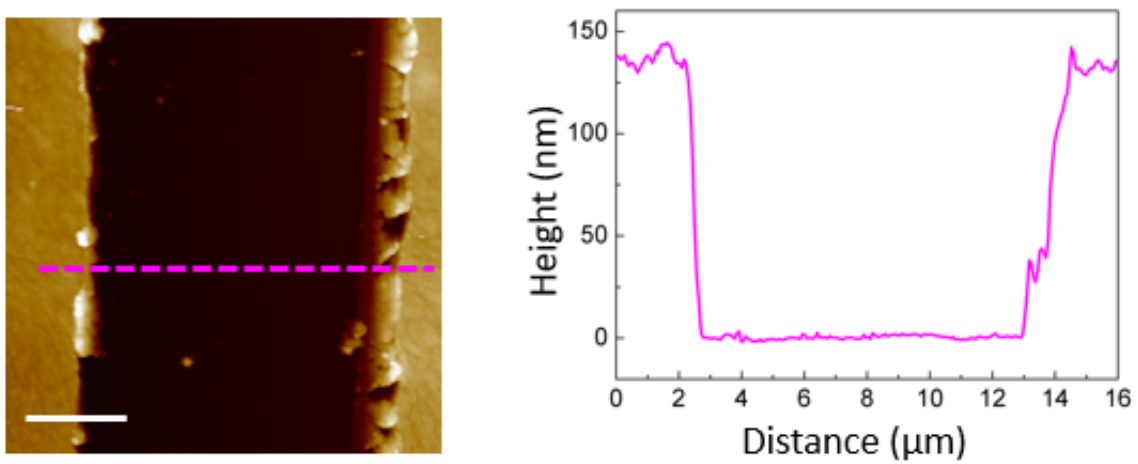

Figure S4. An AFM height image of the scratched region and cross-section analysis showing the height of the film based on P3PHT $(15 \mathrm{mg} / \mathrm{mL})+$ DNA $(3 \mathrm{mg} / \mathrm{mL})$. (Scale bar: $5 \mu \mathrm{m})$ 


\section{S5. XPS measurement}
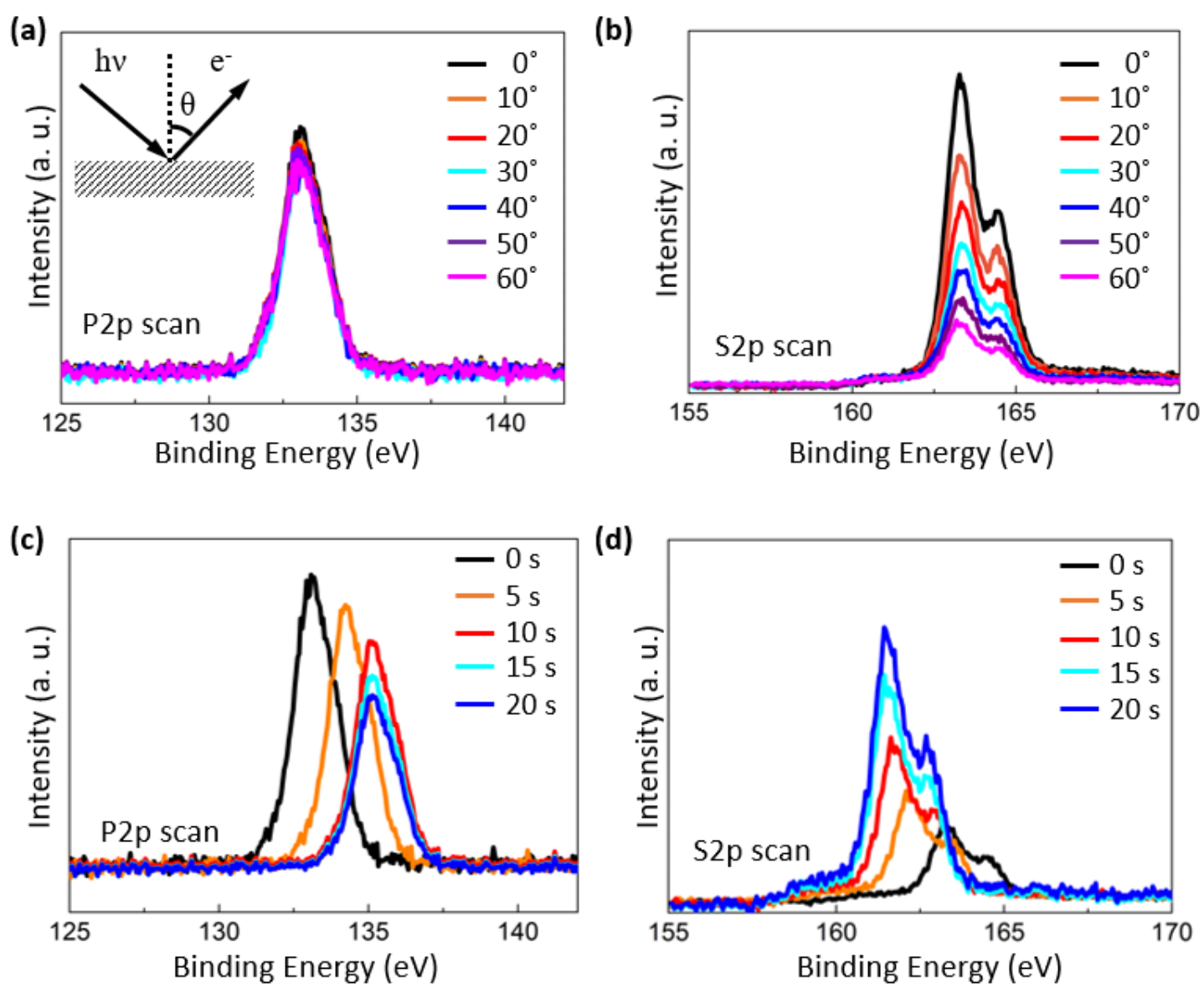

Figure S5. XPS measurements with $h v=0.05 \mathrm{eV}$ photons for angle-dependent emission $\left(0 \sim 60^{\circ}\right)$ of (a) P 2p and (b) S 2p signals and with spin-coated films of P3PHT (15 mg/mL) + DNA (3 $\mathrm{mg} / \mathrm{mL})$. And the depth profiles were measured using argon ion sputtering. The etching condition is ion energy: $3000 \mathrm{eV}$; current: high; raster size: $0.4 \mathrm{~mm}$; etching seep: 200 s/layer. Etching was carefully controlled in $5 \mathrm{~s}$ time steps for 5 total steps. 


\section{S6. Output characteristics for different concentration}
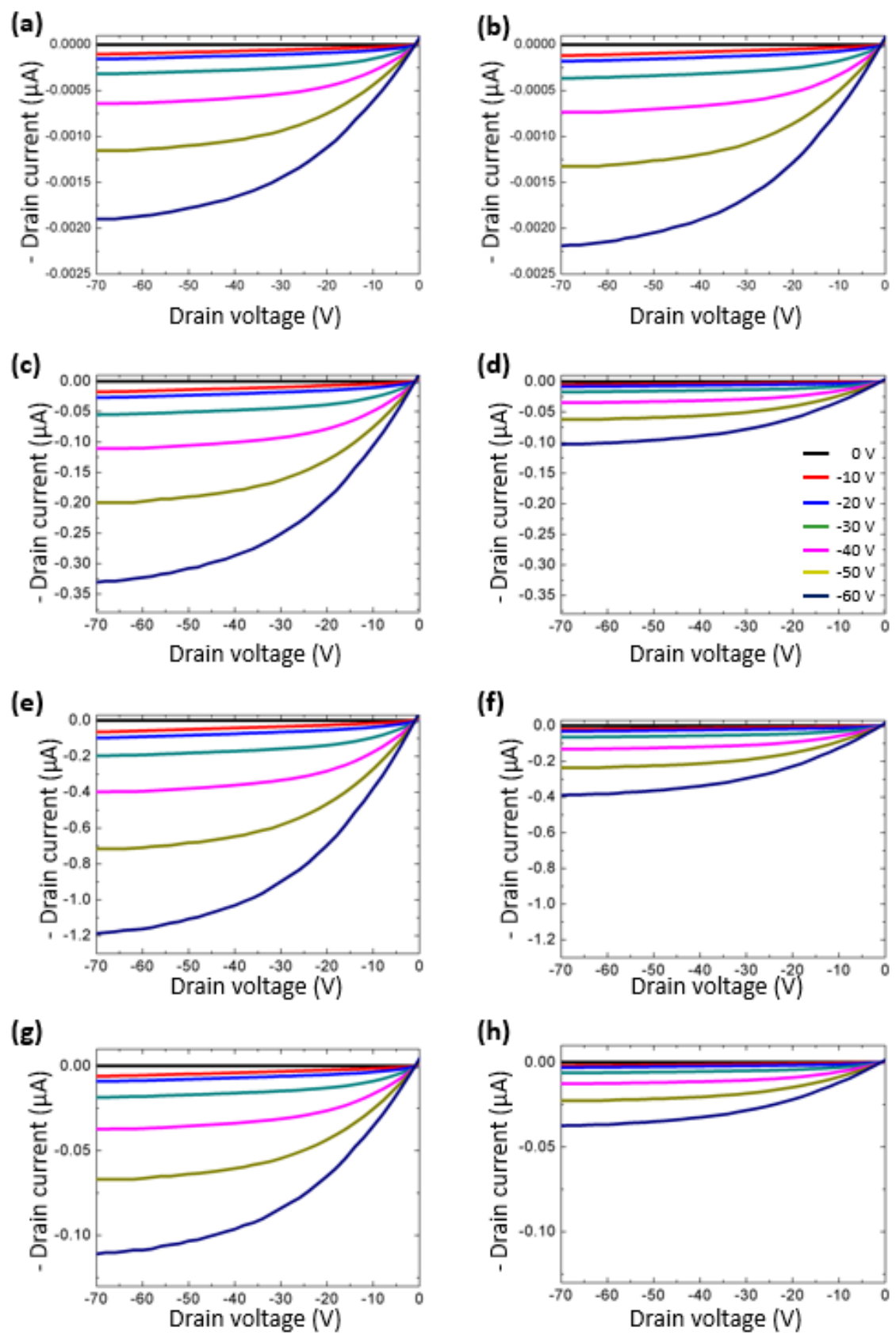

Figure S6. Output characteristics for different concentration condition such as under (a, b) P3PHT (15 mg/mL), (c, d) P3PHT (15 mg/mL) + DNA (1.5 mg/mL), (e, f) P3PHT (15 mg/mL) + DNA $(3 \mathrm{mg} / \mathrm{mL}),(\mathrm{g}, \mathrm{h})$ P3PHT $(15 \mathrm{mg} / \mathrm{mL})+$ DNA $(7.5 \mathrm{mg} / \mathrm{mL})$ decreasing gate voltage $(0 \sim-60 \mathrm{~V}$ with $-10 \mathrm{~V}$ steps) with $(\mathrm{a}, \mathrm{c}, \mathrm{e}, \mathrm{g})$ perpendicular and $(\mathrm{b}, \mathrm{d}, \mathrm{f}, \mathrm{h})$ parallel condition. 


\section{S7. Transfer characteristics of spin-coated films for different concentration condition}

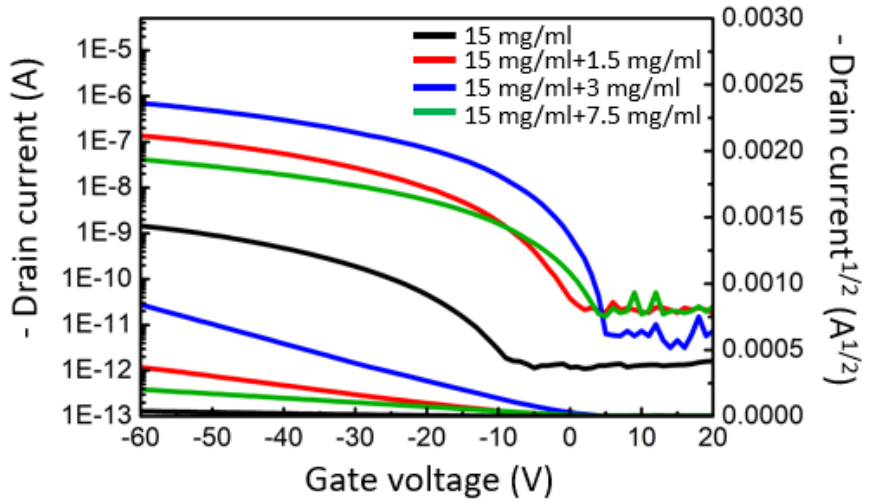

\begin{tabular}{cccc}
\hline Sample & $\begin{array}{c}\text { Mobility } \\
\left(\mathbf{c m}^{2} \mathbf{V}^{-1} \mathbf{s}^{-1}\right)\end{array}$ & $\mathrm{I}_{\text {ON }} / \mathrm{I}_{\text {OFF }}$ & $\begin{array}{c}\mathrm{V}_{\text {th }} \\
(\mathbf{V})\end{array}$ \\
\hline $15 \mathrm{mg} / \mathrm{ml}$ & $(1.25 \pm 0.52) \times 10^{-5}$ & $(8.95 \pm 4.21) \times 10^{2}$ & $-(20 \pm 3.88)$ \\
\hline $15 \mathrm{mg} / \mathrm{ml}+1.5 \mathrm{mg} / \mathrm{ml}$ & $(8.64 \pm 3.2) \times 10^{-4}$ & $(6.35 \pm 3.27) \times 10^{3}$ & $-(5 \pm 2.81)$ \\
\hline $15 \mathrm{mg} / \mathrm{ml}+3 \mathrm{mg} / \mathrm{ml}$ & $(4.1 \pm 1.8) \times 10^{-3}$ & $(1.12 \pm 0.53) \times 10^{5}$ & $-(2 \pm 2.51)$ \\
\hline $15 \mathrm{mg} / \mathrm{ml}+7.5 \mathrm{mg} / \mathrm{ml}$ & $(1.98 \pm 0.83) \times 10^{-4}$ & $(2.35 \pm 1.17) \times 10^{3}$ & $3 \pm 2.11$ \\
\hline
\end{tabular}

Figure S7. Transfer characteristics of spin-coated films for different concentrations such as (a) P3PHT (15 mg/mL), (b) P3PHT (15 mg/mL) + DNA (1.5 mg/mL), (c) P3PHT (15 mg/mL) + DNA (3 mg/mL), (d) P3PHT (15 mg/mL) + DNA (7.5 mg/mL). And standard deviation was obtained from the data of 10 devices. 
S8. Forward and backward transfer curves for spin-coated P3PHT film and P3PHT+DNA film

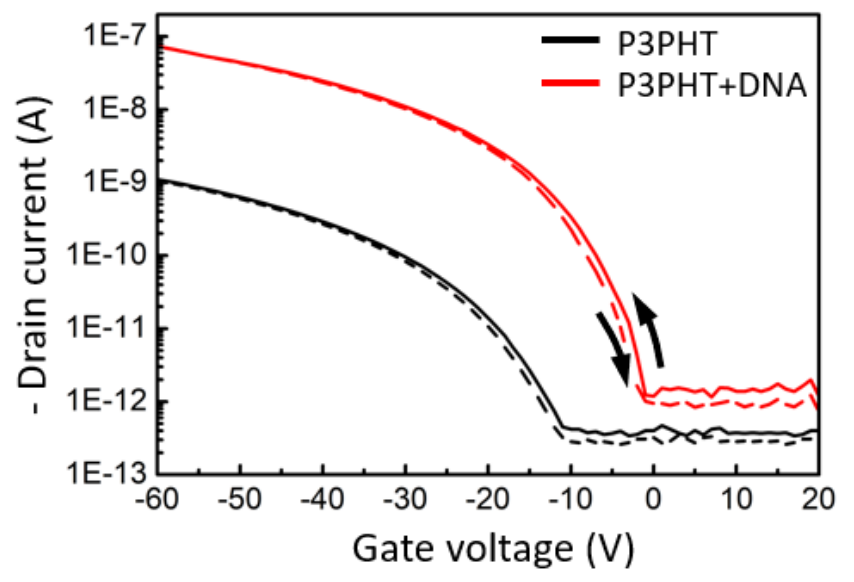

Figure S8. Forward and backward transfer curves for spin-coated film of P3PHT $(15 \mathrm{mg} / \mathrm{mL})$ and P3PHT (15 mg/mL) + DNA (1.5 mg/mL). 


\section{S9. Contact resistance of OFETs}

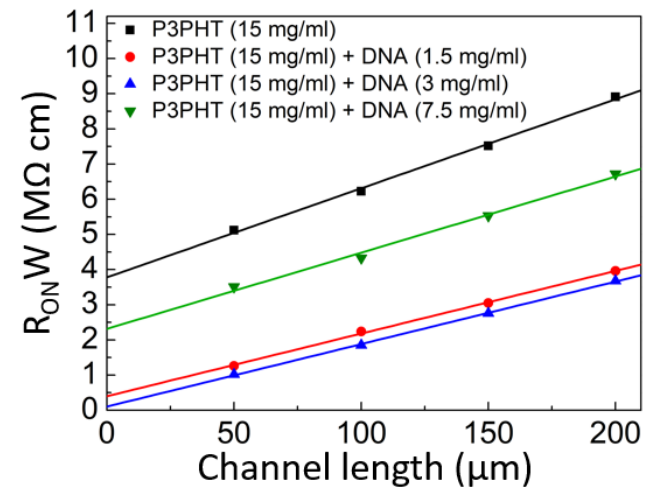

\begin{tabular}{cc}
\hline & $\mathbf{R}_{\text {ON }} \mathbf{W}(\mathbf{M} \Omega \mathrm{cm})$ \\
\hline P3PHT $(15 \mathrm{mg} / \mathrm{ml})$ & 3.78 \\
\hline $\begin{array}{l}\text { P3PHT }(15 \mathrm{mg} / \mathrm{ml}) \\
\text { + DNA }(1.5 \mathrm{mg} / \mathrm{ml})\end{array}$ & 0.4 \\
\hline $\begin{array}{r}\text { P3PHT }(15 \mathrm{mg} / \mathrm{ml}) \\
\text { + DNA }(3 \mathrm{mg} / \mathrm{ml})\end{array}$ & 0.105 \\
\hline $\begin{array}{l}\text { P3PHT }(15 \mathrm{mg} / \mathrm{ml}) \\
\text { + DNA }(7.5 \mathrm{mg} / \mathrm{ml})\end{array}$ & 2.315 \\
\hline
\end{tabular}

Figure S9. Contact resistance of OFETs based on P3PHT $(15 \mathrm{mg} / \mathrm{ml})$ without DNA and with DNA concentration of $1.5 \mathrm{mg} / \mathrm{mL}, 3 \mathrm{mg} / \mathrm{mL}$, and $7.5 \mathrm{mg} / \mathrm{mL}$, respectively at the $\mathrm{V}_{\mathrm{GS}}$ of $-50 \mathrm{~V}$. 
S10. Forward and backward transfer curves of P3PHT, P3PHT $+\mathrm{Cu}^{2+}$, and P3PHT $+\mathrm{Cu}^{2+}$ DNA films

(a)

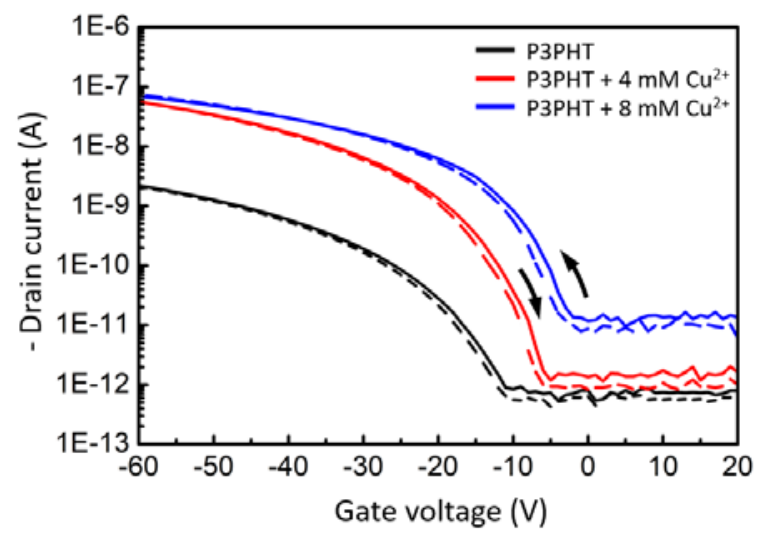

\begin{tabular}{cccc}
\hline Sample & $\begin{array}{c}\text { Mobility } \\
\left(\mathrm{cm}^{2} \mathbf{V}^{-1} \mathbf{s}^{-1}\right)\end{array}$ & $\mathrm{I}_{\text {oN }} / \mathrm{I}_{\text {oFF }}$ & $\begin{array}{c}\mathrm{V}_{\text {th }} \\
(\mathrm{V})\end{array}$ \\
\hline P3PHT $(15 \mathrm{mg} / \mathrm{ml})$ & $(2.1 \pm 0.47) \times 10^{-5}$ & $(2.57 \pm 0.86) \times 10^{3}$ & $-(21 \pm 4.3)$ \\
\hline $\begin{array}{c}\text { P3PHT }(15 \mathrm{mg} / \mathrm{ml}) \\
+4 \mathrm{mM} \mathrm{Cu}\end{array}$ & $(9.3 \pm 3.24) \times 10^{-4}$ & $(6.14 \pm 3.24) \times 10^{4}$ & $-(13 \pm 3.8)$ \\
\hline $\begin{array}{c}\text { P3PHT }(15 \mathrm{mg} / \mathrm{ml}) \\
+8 \mathrm{mM} \mathrm{Cu}\end{array}$ & $(7.8 \pm 2.73) \times 10^{-4}$ & $(7.82 \pm 3.95) \times 10^{3}$ & $-(6 \pm 1.8)$ \\
\hline
\end{tabular}

(b)

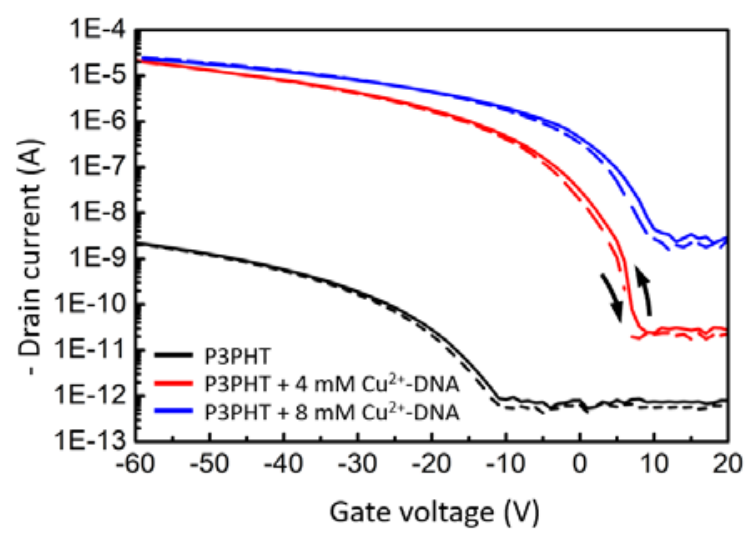

\begin{tabular}{cccc}
\hline Sample & $\begin{array}{c}\text { Mobility } \\
\left(\mathrm{cm}^{2} \mathbf{V}^{-1} \mathrm{~s}^{-1}\right)\end{array}$ & $\mathrm{I}_{\text {oN }} / \mathrm{I}_{\text {OFF }}$ & $\begin{array}{c}\mathrm{V}_{\text {th }} \\
\text { (V) }\end{array}$ \\
\hline P3PHT $(15 \mathrm{mg} / \mathrm{ml})$ & $(2.1 \pm 0.47) \times 10^{-5}$ & $(2.57 \pm 0.86) \times 10^{3}$ & $-(21 \pm 4.3)$ \\
\hline $\begin{array}{c}\text { P3PHT }(15 \mathrm{mg} / \mathrm{ml}) \\
+4 \mathrm{mM} \mathrm{Cu}{ }^{2+}-\mathrm{DNA}(1.5 \mathrm{mg} / \mathrm{ml})\end{array}$ & $0.22 \pm 0.085$ & $(4.04 \pm 2.29) \times 10^{5}$ & $5 \pm 2.15$ \\
\hline $\begin{array}{c}\text { P3PHT }(15 \mathrm{mg} / \mathrm{ml}) \\
+8 \mathrm{mM} \mathrm{Cu}\end{array}$ & $0.20 \pm 0.072$ & $(5.47 \pm 2.49) \times 10^{2+}$-DNA $(1.5 \mathrm{mg} / \mathrm{ml})$ & $10 \pm 3.52$ \\
\hline
\end{tabular}

Figure S10. Forward and backward transfer curves for shear aligned (a) P3PHT and P3PHT + $\mathrm{Cu}^{2+}$ and (b) P3PHT and P3PHT $+\mathrm{Cu}^{2+}$-DNA films with different $\mathrm{Cu}^{2+}$ concentration. The sourcedrain channel direction was perpendicular to the shear direction. 